\title{
Incidence of dilated cardiomyopathy
}

\author{
Walter H. Abelmann
}

Charles A. Dana Research Institute and the Harvard-Thorndike Laboratory of Beth Israel Hospital, Department of Medicine, Beth Israel Hospital and Harvard Medical School, Boston, Mass., USA.

\begin{abstract}
Summary: Full reliable data on the incidence and prevalence of dilated cardiomyopathy are not available. In the United States, at least $0.7 \%$ of cardiac deaths are attributable to cardiomyopathy. Dilated cardiomyopathy probably contributes the great majority of these cases. The mortality rate for cardiomyopathy in males is twice that of females, and for blacks it is 2.4 times that of whites. Cardiomyopathy was diagnosed in $0.67 \%$ of patients discharged from hospitals in 1979 with diagnoses of disease of the circulatory system. Cardiomyopathy accounted for $1 \%$ of general cardiologists' and for $7 \%$ of academic cardiologists' patient encounters.

In Scandinavia, population surveys suggested an annual incidence of dilated cardiomyopathy ranging from 0.73 to 7.5 cases per 100,000 population; for Tokyo this figure is 2.6 . The prevalence of cardiomyopathy in underdeveloped and in tropical countries is considerably higher than in developed countries.
\end{abstract}

The true incidence and prevalence of dilated cardiomyopathy are unknown and likely to remain unknown for some time to come. There are several reasons for this state of affairs, which also apply to several other disease syndromes of unknown or uncertain aetiology, the definition, recognition and understanding of which are in a state of change.

(1) The clinical diagnosis is unreliable (Abelmann, 1984). Dilated cardiomyopathy is generally underdiagnosed, especially in the earlier, asymptomatic stages. Inasmuch as the incidence is greater in males, and especially in mature males (see below), it is often misdiagnosed as ischaemic heart disease (especially in developed countries), sometimes as valvular heart disease, and, in certain countries of South America, as Chagas' disease.

(2) Autopsies are performed only occasionally. Autopsies are carried out in only a small fraction of deaths from heart disease, and, at least in the United States, only on a fraction of sudden deaths.

(3) Samples of the population are usually biased. Clinical as well as post-mortem series are usually reported from centres with special interest, especially high incidence, or both.

(4) Differentiation between different types of cardiomyopathy is often not clear. The current (1980) WHO definition of cardiomyopathy as heart muscle disease of unknown aetiology is more narrow than the original WHO (Fejfar, 1968) definition of cardiomyopathy. Inasmuch as the clinical syndromes and natural history of cardiomyopathies of known and of unknown cause

Correspondence: W.H. Abelmann, M.D. may be very similar and diagnostic classifications used locally may not distinguish between them, the available data are more likely to approximate the earlier, broader WHO definition of cardiomyopathy as conditions of different, frequently unknown aetiology in which the dominant feature is cardiomegaly and cardiac failure, exclusive of myocardial dysfunction due to valvular disease, coronary artery disease, systemic or pulmonary vascular disease (Fejfar, 1968).

(5) Apparent changes in incidence and prevalence of cardiomyopathy must be interpreted with caution. In recent years, the incidence of cardiomyopathy appears to have increased. While this impression is difficult to refute, changing frequencies of encounters are more likely to be a function of the tremendous increase in the sensitivity of non-invasive and invasive methods of detection of impaired myocardial function, the expansion of the number of physicians trained in diagnostic cardiology, and changing patient-referral patterns.

With these caveats in mind, let us examine some of the available data.

\section{Mortality from cardiomyopathy}

In 1979, deaths from all disease of the circulatory system in the United States numbered 963,037. There were 6,912 reported deaths from cardiomyopathy, or $0.71 \%$ of all cardiovascular deaths (Vital Statistics of the U.S., 1979). This should be accepted as a minimal number, probably a significant underestimate. The 
distribution of these deaths by sex is significantly and strikingly uneven, 4,669 deaths occurring in males and only 2,243 in females, representing 0.94 and $0.48 \%$ of all cardiovascular deaths, respectively. The mortality from cardiomyopathy is also strikingly greater in blacks than in whites, 1,420 of a total of 94,501 cardiovascular deaths $(1.51 \%)$ versus 5,416 of 862,872 $(0.63 \%)$, respectively.

\section{Prevalence of cardiomyopathy in hospitals and physicians' offices}

The US Hospital Discharge Survey in 1979 provides data which represent extrapolation from some $\mathbf{4 0 0}$ monitored institutions to the country as a whole. Of $18,346,000$ patients diagnosed as having diseases of the circulatory system, 126,000 or $0.67 \%$ had cardiomyopathy. The respective figures for the primary diagnosis were $5,488,000$ and $46,000(0.84 \%)$. A similar prevalence was found by a survey of US cardiology manpower in 1973 (Swan \& Gifford, 1974): cardiomyopathy accounted for $1 \%$ of all cardiologists' patient encounters. In academic cardiologists' practices, however, the prevalence was $7 \%$.

\section{Incidence of cardiomyopathy in population surveys}

Torp $(1978,1981)$ surveyed the incidence of congestive (dilated) cardiomyopathy in the city of Malmö, Sweden, which has a relatively stable population of 250,000 and is served by only one hospital with an autopsy rate of $90 \%$. Over the 10 year period of this prospective study, from 1970 to 1979,89 cases of dilated cardiomyopathy were diagnosed clinically, and an additional 56 cases at post-mortem examina-

\section{References}

ABELMANN, W.H. (1984). Classification and natural history of primary myocardial disease. Progress in Cardiovascular Disease, 27, 73.

BAGGER, J.P., BAANDRUP, V., RASMUSSEN, K., MOLLER, M. \& VESTERLUND, T. (1984). Cardiomyopathy in Western Denmark. British Heart Journal, 52, 327.

CORREA, P., RESTREPO, C., GARCIA, C. \& QUIROZ, A.C. (1963). Pathology of heart diseases of undetermined etiology which occur in Cali, Colombia. American Heart Journal, 66, 584.

FEJFAR, Z. (ed.) (1968). Accounts of International Meetings. Idiopathic cardiomyopathy. Bulletin of the WHO, 38, 979.

KAWAI, C. (ed.) (1978). Idiopathic cardiomyopathy. Naika Series No. 32, Nankodo: Tokyo.

KAWAI, C. (1980). In Cardiomyopathy, Sekiguchi, M. \& Olsen, E.G.J. (eds). p. 3. University of Tokyo Press: Tokyo.

KOBERLE, F. (1957). Die chronische Chagaskardiopathie. Virchow's Archiv, 330, 267. tion, using the current WHO/ISFC classification (1980). Thus there was an incidence of 7.5 cases of dilated cardiomyopathy per 100,000 inhabitants per year. It is of interest that another, albeit retrospective, Scandinavian study (Bagger et al., 1984) yielded a significantly lower annual incidence of 0.73 cases of dilated cardiomyopathy per 100,000 population in $1980 / 1$, based upon a population of $2,798,000$ in Western Denmark. The authors considered this figure to be a minimum, and gave several reasons for this being an underestimate, including rigid criteria, short period of observation, low autopsy rate and incomplete referrals.

A survey by questionnaire in 1974 revealed a minimum annual incidence of 2.6 cases of cardiomyopathy (all types) per 100,000 population in Tokyo (Kawai, 1978), of which, however, only about $38 \%$ had dilated cardiomyopathy (Kawai, 1980). In contrast, in the United States, dilated cardiomyopathy comprises about $90 \%$ of all cardiomyopathies.

It is noteworthy that the above data were all obtained in developed countries. It is generally agreed that in many underdeveloped countries, especially in the tropics, dilated cardiomyopathy, including specific heart muscle diseases such as Chagas' disease (Correa et al., 1963) and myocarditis, are considerably more frequent than in developed countries, ranging from $3 \%$ of cardiac diagnoses at autopsy in Cali, Colombia (Correa et al., 1963), to $45 \%$ of all autopsies in Bahia Brazil (Köberle, 1957).

\section{Editorial note}

A recent study in England revealed an overall point prevalence of 8.317 cases per 100,000 of the population [William, D.G. \& Olsen, E.G.J. (1985). Prevalence of overt dilated cardiomyopathy in two regions of England. British Heart Journal, 54, 153].

SWAN, H.J.C. \& GIFFORD, R.W. (1974). Current profile of the professional activities of the American cardiologist. American Journal of Cardiology, 34, 417.

TORP, A. (1978). Incidence of congestive cardiomyopathy. Postgraduate Medical Journal, 54, 435.

TORP, A. (1981). In Congestive Cardiomyopathy, Goodwin, J.F., Hjalmarson, A. \& Olsen, E.C.J. (eds). p. 18. AB Hässle: Mölndal, Sweden.

U.S. Hospital Discharge Survey 1979. U.S. Dept. Health and Human Services, Public Health Service, National Center for Health Statistics, Hyattsville, Maryland, 1984.

Vital Statistics of the U.S. 1979, Vol II, Part A. U.S. Dept. Health and Human Services, Public Health Service, National Center for Health Statistics, Hyattsville, Maryland, 1984.

WHO/ISFC CLASSIFICATION: Report of the WHO/ISFC task force on the definition and classification of cardiomyopathies. (1980). British Heart Journal, 44, 672. 